\title{
Using the mood disorder questionnaire and bipolar spectrum diagnostic scale to detect bipolar disorder and borderline personality disorder among eating disorder patients
}

Toshihiko Nagata $^{1 *}$, Hisashi Yamada ${ }^{2}$, Alan R Teo ${ }^{3}$, Chiho Yoshimura ${ }^{2}$, Yuya Kodama ${ }^{1}$ and Irene van Vliet ${ }^{4}$

\begin{abstract}
Background: Screening scales for bipolar disorder including the Mood Disorder Questionnaire (MDQ) and Bipolar Spectrum Diagnostic Scale (BSDS) have been plagued by high false positive rates confounded by presence of borderline personality disorder. This study examined the accuracy of these scales for detecting bipolar disorder among patients referred for eating disorders and explored the possibility of simultaneous assessment of co-morbid borderline personality disorder.
\end{abstract}

Methods: Participants were 78 consecutive female patients who were referred for evaluation of an eating disorder. All participants completed the mood and eating disorder sections of the SCID-I/P and the borderline personality disorder section of the SCID-II, in addition to the MDQ and BSDS. Predictive validity of the MDQ and BSDS was evaluated by Receiver Operating Characteristic analysis of the Area Under the Curve (AUC).

Results: Fifteen (19\%) and twelve (15\%) patients fulfilled criteria for bipolar II disorder and borderline personality disorder, respectively. The AUCs for bipolar II disorder were 0.78 (MDQ) and 0.78 (BDSD), and the AUCs for borderline personality disorder were 0.75 (MDQ) and 0.79 (BSDS).

Conclusions: Among patients being evaluated for eating disorders, the MDQ and BSDS show promise as screening questionnaires for both bipolar disorder and borderline personality disorder.

Keywords: Bipolar disorder, Borderline personality disorder, Eating disorder, Comorbidity, Screening scale

\section{Background}

As bipolar disorders are serious mental disorders that can cause severe lifelong functional impairment, early recognition of the diagnosis and early introduction of mood stabilizers are crucial for improvement of outcomes [1]. Nonetheless, most patients with bipolar disorder go years before receiving an appropriate diagnosis and starting mood stabilizers [1]. Borderline personality disorder is the most common personality disorder in clinical settings, and causes marked distress and impairment in social, occupational, and role functioning [2]. Yet, similar to bipolar disorder, borderline personality disorder is often

\footnotetext{
* Correspondence: TOSHI@med.osaka-cu.ac.jp

'Department of Neuropsychiatry, Osaka City University Graduate School of Medicine, 1-4-3 Asahimachi, Abunoku, Osaka 545-8585, Japan

Full list of author information is available at the end of the article
}

incorrectly diagnosed or underdiagnosed in clinical practice [2]. Both bipolar and borderline personality disorders are associated with high rates of completed suicide $[1,2]$ and are common among patients with mood disorders $[1,2]$ and eating disorders $[3,4]$.

Recently, the boundary of these disorders has been a focus of debate [5-9]. There are two viewpoints about the relationship between bipolar disorder, especially bipolar II disorder, and borderline personality disorder. The first one is that underlying cyclothymic temperament can explain the relationship, and borderline personality disorder [9] (as well as bulimia nervosa [8]) are variants of bipolar disorders. Others insist that there is clear boundary between bipolarity and borderline personality disorder, and they resist the expansion of bipolar 
disorder as an invasion of "bipolar imperialism" toward other diagnostic categories [5].

Two screening scales, the Mood Disorder Questionnaire (MDQ) [10] and Bipolar Spectrum Diagnostic Scale (BSDS) [11], have been developed to ameliorate the underdiagnosis of bipolar disorders. These instruments show good psychometric properties to detect bipolarity among patients with unipolar depression and are recommended as screening tools for bipolar disorders among patients with unipolar depression [1]. However, Zimmerman et al. (2011) [12,13] reported that the false positive rates of these two scales were not negligible because of the symptomatic overlap of bipolar disorder and other conditions [1]. Patients diagnosed with bipolar disorder by previous doctors were significantly more likely to be diagnosed with borderline personality disorder compared to patients who were not diagnosed with bipolar disorder $(24.4 \%$ vs. $6.1 \%)$ [14]. Viewed another way, these results suggest the possibility that both bipolar and borderline personality disorders can be simultaneously detected by these scales.

To the best of our knowledge, the MDQ and BSDS have never been used to detect bipolar disorders or borderline personality disorder among eating disorder patients, despite the relatively high comorbidity rates of these disorders $[3,4]$. In contrast to common attention towards impulsivity and borderline personality disorder among eating disorder patients [4], the presence of comorbid bipolar disorder has rarely received attention of eating disorder specialists [15]. However, some evidence suggests increased prevalence of bipolar II disorder [16], ego-syntonic hypomania may escape clinical detection, and comorbid bipolar disorder requires special therapeutic considerations [3]. Thus, screening scales for bipolar disorder might be more important than eating disorder specialists traditionally thought.

The aim of the current study was to examine the diagnostic accuracy (including sensitivity and specificity) of the MDQ and BSDS to detect bipolar disorders among patients that were referred for evaluation of an eating disorder. We also explored the possibility that the two scales can detect borderline personality disorder among this population. We hypothesized that the diagnostic accuracy of the two screening tests (MDQ and BSDS) for borderline personality disorder might be similar to that for bipolar disorders.

\section{Methods}

\section{Participants}

Participants were recruited from a consecutive series of ninety female patients who were referred for evaluation and/or treatment of an eating disorder to the first and second authors (T. N. and H. Y.) at the Department of Neuropsychiatry, Osaka City University Hospital from February to June 2011. To maximize real world applicability of
Table 1 Demographic and clinical characteristics of participants $(n=78)$ with eating disorders

\begin{tabular}{ll}
\hline & Mean (SD) or N (\%) \\
\hline Age, years & $29.5(7.4)$ \\
Education, years & $13.8(2.4)$ \\
Marital status, single & $58(74 \%)$ \\
Occupational status & \\
Unemployed & $37(47 \%)$ \\
Part-time worker (or student) & $16(21 \%)$ \\
Full-time worker (or student) & $25(32 \%)$ \\
Age at onset of eating disorder & $19.1(4.8)$ \\
Body mass index & $17.3(4.4)$ \\
Subtype of eating disorder & \\
Anorexia nervosa restricting type & $11(14 \%)$ \\
Anorexia nervosa binge-eating purging subtype & $24(31 \%)$ \\
Bulimia nervosa purging subtype & $27(35 \%)$ \\
Restricting EDNOS & $2(3 \%)$ \\
Binge-eating / purging EDNOS & $14(18 \%)$ \\
Frequency of binge eating (episodes/week) & $4.6(4.6)$ \\
Frequency of vomiting (episodes/week) & $4.9(4.2)$ \\
History of major depressive episode(s) & $55(71 \%)$ \\
Age at onset, years & $20.5(4.8)$ \\
History of manic episode(s) & $0(0 \%)$ \\
History of hypomanic episode(s) & $15(19 \%)$ \\
Age at onset, years & $23.1(5.6)$ \\
Borderline personality disorder & $12(15 \%)$ \\
Histrionic personality disorder & $23(30 \%)$ \\
BSDS total score & $9.2(6.2)$ \\
MDQ $\geq 7$ with moderate functional impairment & $22(28 \%)$ \\
\hline MDQ: & $31(40 \%)$ \\
Bith minor functional impairment & $19 \%)$ \\
\hline
\end{tabular}

MDQ: Mood Disorder Questionnaire, BSDS: Bipolar Spectrum Diagnostic Scale, EDNOS: Eating disorder not otherwise specified.

findings, exclusion criteria were minimal: 1) substance use disorder requiring acute detoxification (if such cases hospital receptionists recommended patients to visit an alcohol treatment facility), 2) self-reported history of schizophrenia, schizoaffective disorder, schizophreniform disorder, or organic mental illnesses, as determined by the screening questionnaire of the SCID-I/P, or 3) inability or unwillingness to complete self-rating scales. The patients with eating disorder not otherwise specified (EDNOS) were not excluded as prior research has shown these patients often are referred for eating disorder treatment and may have comorbid bipolar or borderline personality disorder [17] . Of the ninety patients who were screened, seventy- 


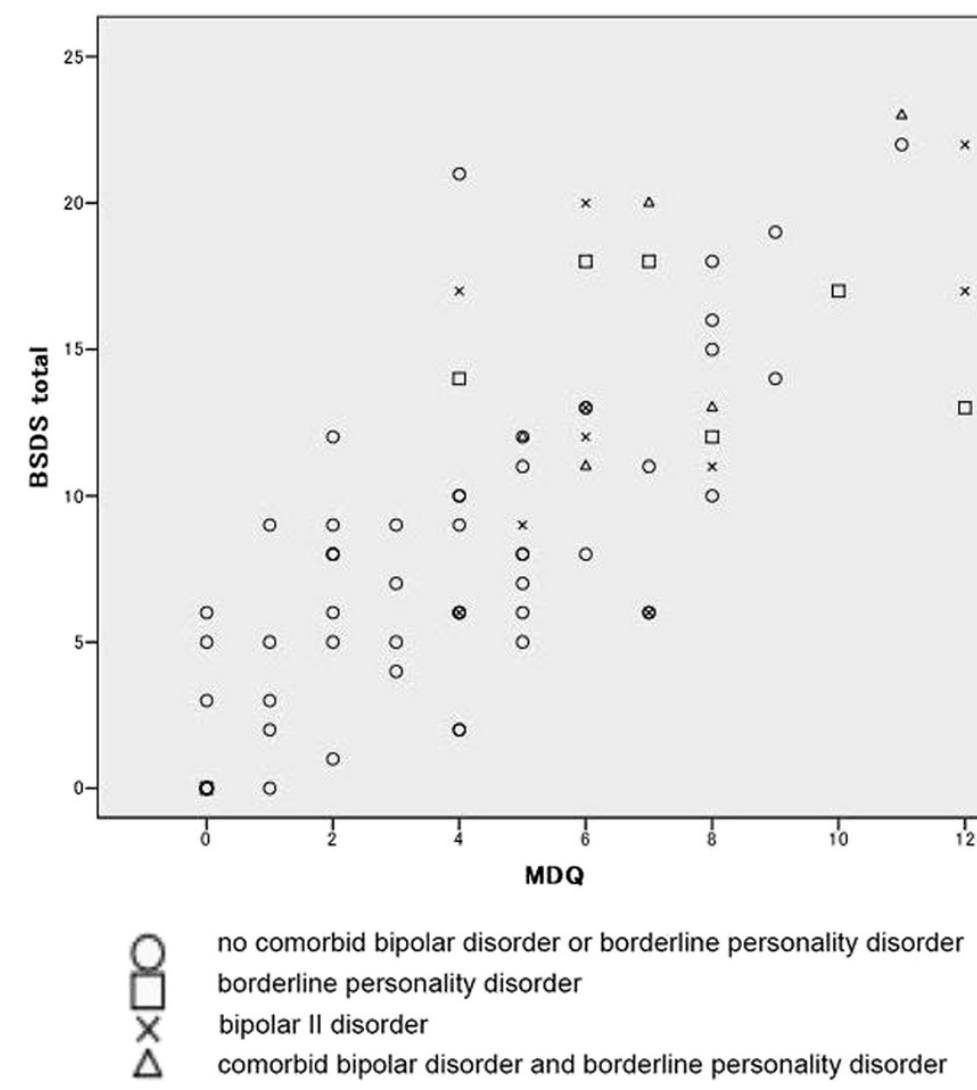

Figure 1 Diagnoses of patients $(\mathbf{n}=\mathbf{7 8})$ based on MDQ and BSDS scores. MDQ: Mood Disorder Questionnaire, BSDS: Bipolar Spectrum Diagnostic Scale.

eight patients enrolled in the study, and all enrollees completed the study. Of the twelve patients that did not participate in the study, six deemed their psychiatric problem minor and opted to cope with the difficulty, four were reluctant to undergo detailed psychiatric assessment, and two declined to participate for unknown reasons. As part of routine clinical care, patients received cognitive behavioral therapy, dialectical behavioral therapy, or medication management depending on the results of their assessment, even when their provisional primary diagnosis (defined as the disorder most influencing their global functioning) was other than an eating disorder. All patients provided written informed consent before entering the study. This study was approved by the institutional review committee of the Osaka City University Graduate School of Medicine.

\section{Measurements}

Two self-report screening scales for bipolar disorder, the Mood Disorder Questionnaire (MDQ) $[10,18]$ and Bipolar Spectrum Diagnostic Scale (BSDS) [11,19], were competed by all participants. The MDQ screens for a lifetime history of mania or hypomania using 13 dichotomous (yes/no) symptom questions reflecting the DSM-IV inclusion criteria. The symptom questions are followed by a single question about whether the symptoms clustered during the same period. The final question evaluates the level of impairment resulting from the symptoms on a 4-point scale (no, minor, moderate, or serious problems). A score of 7 or more on the first 13 items, "yes" to symptom clustering, and moderate or greater problems was proposed as the cut-off level in the original study [10]. In a Japanese study of unipolar depressive patients, a lower cut-off of more than 5 with minor or greater problems was proposed [19]. The BSDS was developed to target bipolar II disorder and bipolar disorder not otherwise specified and supplement clinicians' semi-structured interviews [11]. The BSDS consists of two parts: first, a paragraph containing 19 statements describing many of the symptoms of bipolar disorder, and, second, a single multiple-choice question asking respondents how well the paragraph describes them. The total score ranges from 0 to 25. A score of 13 for the original version [11], 12 for the Chinese version [20], and 11 for the Japanese version [19] have been proposed as cut-off points.

All participants underwent a direct (face-to-face) assessment conducted by T. N. or H. Y. who each have more than ten years' experience treating eating disorders. This assessment included the mood and eating disorder 
Table 2 Sensitivity and specificity of the MDQ and BSDS to diagnose bipolar disorder, borderline personality disorder, and histrionic personality disorder according to several cut-off points

\begin{tabular}{|c|c|c|c|}
\hline & BPII & BPD & Histrionic \\
\hline & \multicolumn{3}{|c|}{ N (sensitivity, specificity, LR+) } \\
\hline & 15 & 12 & 23 \\
\hline $\mathrm{MDQ} \geq 7$ with moderate functional impairment & $3(0.20,0.81,1.1)$ & $5(0.42,0.85,2.8)$ & $6(0.26,0.84,1.6)$ \\
\hline $\mathrm{MDQ} \geq 6$ with moderate functional impairment & $8(0.53,0.78,2.4)$ & $7(0.58,0.80,2.9)$ & $7(0.30,0.76,2.1)$ \\
\hline$M D Q \geq 6$ with minor functional impairment & $8(0.53,0.78,2.4)$ & $7(0.58,0.77,2.5)$ & $8(0.35,0.75,1.4)$ \\
\hline$M D Q \geq 5$ with minor functional impairment & $10(0.67,0.68,2.1)$ & $8(0.67,0.67,2.0)$ & $12(0.52,0.67,1.6)$ \\
\hline$B S D S \geq 13$ & $8(0.53,0.78,2.4)$ & $8(0.67,0.79,3.2)$ & $10(0.44,0.78,2.0)$ \\
\hline $\mathrm{BSDS} \geq 12$ & $10(0.67,0.73,2.9)$ & $10(0.83,0.74,3.2)$ & $12(0.52,0.73,1.9)$ \\
\hline$B S D S \geq 11$ & $12(0.80,0.70,2.7)$ & $11(0.92,0.70,3.1)$ & $15(0.65,0.71,2.2)$ \\
\hline $\mathrm{BSDS} \geq 10$ & $12(0.80,0.64,2.2)$ & $11(0.92,0.64,2.6)$ & $15(0.65,0.64,1.8)$ \\
\hline \multirow[t]{4}{*}{$B S D S \geq 9$} & $13(0.88,0.59,2.2)$ & $11(0.92,0.58,2.2)$ & $18(0.78,0.82,4.3)$ \\
\hline & BPII only & BPD only & \\
\hline & \multicolumn{2}{|c|}{ N (sensitivity, specificity, LR+) } & \\
\hline & 10 & 7 & \\
\hline $\mathrm{MDQ} \geq 7$ with moderate functional impairment & $2(0.20,0.81,1.1)$ & $4(0.57,0.78,2.6)$ & \\
\hline $\mathrm{MDQ} \geq 5$ with minor functional impairment & $7(0.70,0.66,2.1)$ & $5(0.71,0.65,2.0)$ & \\
\hline$B S D S \geq 13$ & $5(0.50,0.75,2.0)$ & $5(0.71,0.70,2.4)$ & \\
\hline$B S D S \geq 12$ & $6(0.60,0.69,1.9)$ & $6(0.86,0.70,2.9)$ & \\
\hline$B S D S \geq 11$ & $7(0.70,0.67,2.1)$ & $6(0.86,0.65,2.5)$ & \\
\hline
\end{tabular}

MDQ: Mood Disorder Questionnaire, BSDS: Bipolar Spectrum Diagnostic Scale, BPIl: bipolar II disorder, BPII only: bipolar II disorder without borderline personality disorder, $B P D$ : borderline personality disorder, $B P D$ only: borderline personality disorder without bipolar II disorder, Histrionic; Histrionic personality disorder, $L R+$ : Likelihood Ratio for a positive test.

sections of the Structured Clinical Interview for DSM-IV, (SCID-I/P) [21,22], and the borderline and histrionic personality sections of the Structured Clinical Interview for DSM-IV Personality Disorders (SCID-II) [23,24]. These limited portions of the SCID-II were selected because our previous study found that the prevalence of other personality disorders such as antisocial and narcissistic personality disorder were low $(0$ and 3\%, respectively) in our setting [25] while previous studies $[7,26]$ have suggested a relationship between bipolarity and histrionic personality disorder.

\section{Statistical analysis}

We examined the diagnostic accuracy of the MDQ and BSDS for bipolar as well as personality disorders, using the SCID-I and II as the gold standard diagnostic tool. Sensitivity, specificity and likelihood ratio for a positive test $[\mathrm{LR}+$, sensitivity/(1-specificity)] [27] were calculated
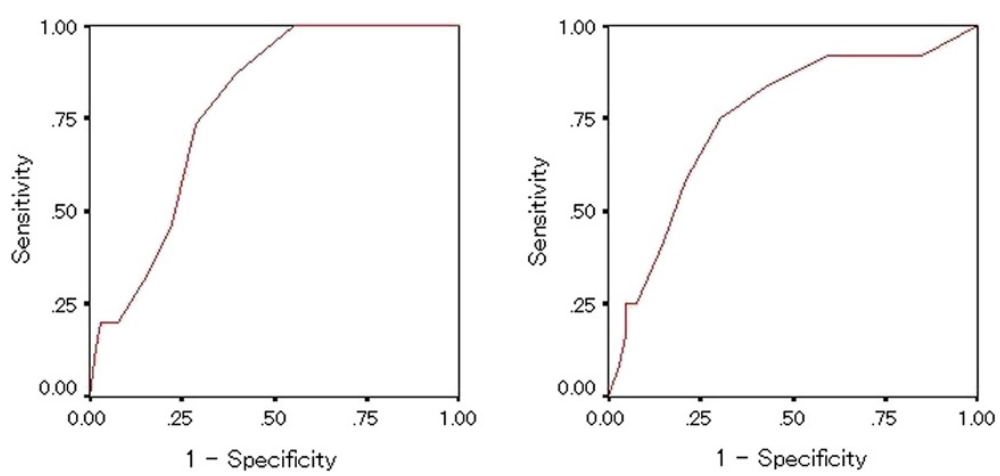

Figure 2 Receiver Operating Characteristic (ROC) curve of diagnostic accuracy of score on question one of the mood disorder questionnaire; Bipolar II disorder (left) and borderline personality disorder (right). 

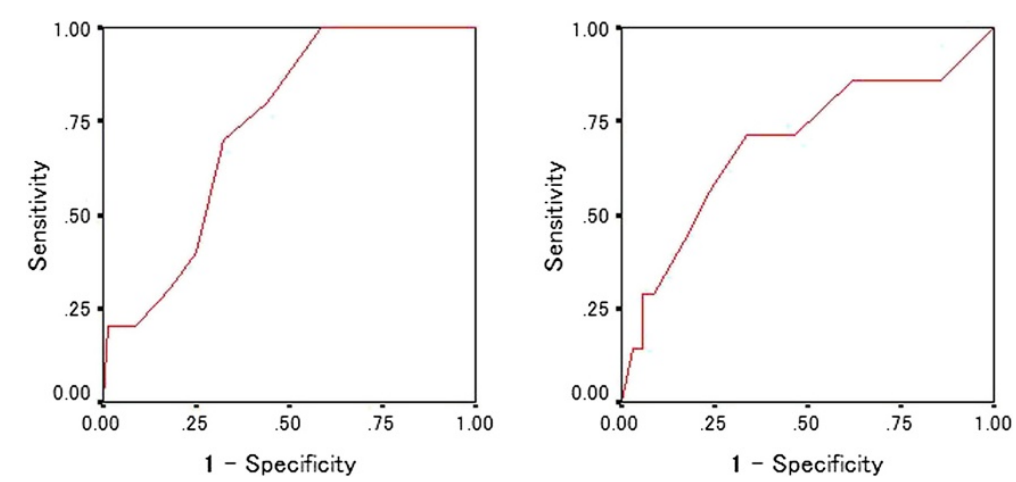

Figure 3 Receiver Operating Characteristic (ROC) curve of diagnostic accuracy of score on the mood disorder questionnaire; Bipolar II disorder without borderline personality disorder (left) and borderline personality disorder without bipolar II disorder (right).

according to several cut-off points suggested by previous studies [10,11,18-20]. To the best of our knowledge, no previous study has explored the possibility that the two scales might detect borderline or histrionic personality disorders. Given this, we used the same cut-off point to detect the personality disorders as for bipolar disorder. In addition, the Receiver Operating Characteristic (ROC) curve and Area Under the Curve (AUC) of both scales for bipolar and personality disorders were calculated. AUC is a preferred measure of accuracy as it is uninfluenced by prevalence, which would be expected to be higher in this study's patient population. All data were analyzed with SPSS 16.0 (SPSS, Inc., Chicago in USA).

\section{Results}

The patients' demographic and clinical characteristics are depicted in Table 1. A high level of functional impairment was suggested by the high rate of single participants (around two-thirds), unemployment (around half) and chronicity (around ten years).

Fifty-five (71\%) had a lifetime history of a major depressive episode and 15 (19\%) a hypomanic episode (bipolar II disorder). No patients had bipolar I disorder. Twelve
(15\%) had borderline personality disorder and 23 (29\%) histrionic personality disorder. Five (6\%) had both lifetime bipolar II disorder and borderline personality disorder. Similarly, eight (10\%) had both lifetime bipolar II disorder and histrionic personality disorder. No patients with anorexia nervosa restricting subtype or restricting EDNOS had bipolar, borderline, or histrionic personality disorders.

Figure 1 shows the monomodal distribution (rather than bimodal) of patients' score on the MDQ and BSDS. As Table 2 shows, both the MDQ and BSDS exhibited similar sensitivity, specificity, and likelihood ratios (LR+) for detecting bipolar II disorder. Further, these two scales showed comparable accuracy in detecting borderline personality disorder. Since accuracy for detecting histrionic personality disorder was relatively low, just bipolar II disorder and borderline personality disorder were the focus of the following analyses. Results were similar for detecting bipolar II disorder without comorbid borderline personality disorder and borderline personality disorder without comorbid bipolar II disorder.

To evaluate the ability to detect bipolar II disorders, ROC curves and the AUCs (95\% Confidence Interval) of the MDQ (Figure 2) and BSDS (Figure 3) were calculated.
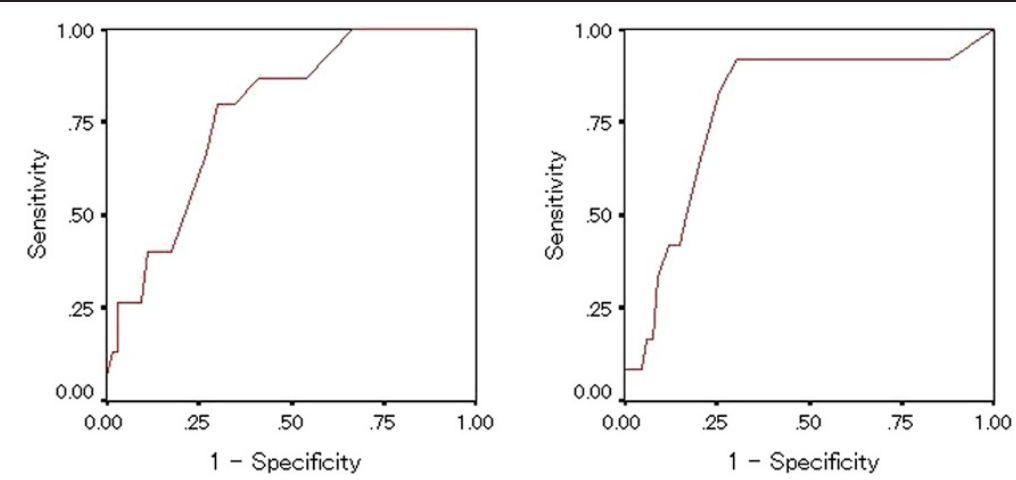

Figure 4 Receiver Operating Characteristic (ROC) curve of diagnostic accuracy of score on question one of the bipolar spectrum diagnostic scale; Bipolar II disorder (left) and borderline personality disorder (right). 

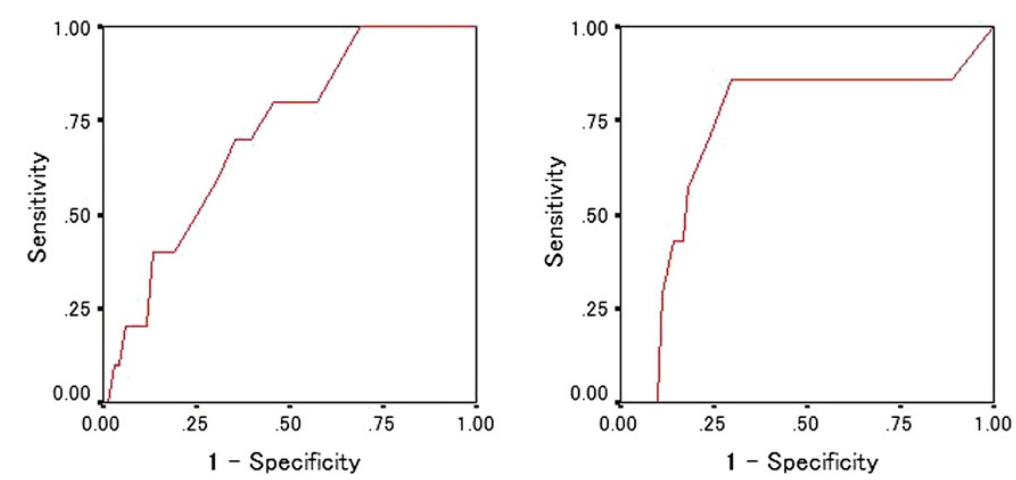

Figure 5 Receiver Operating Characteristic (ROC) curve of diagnostic accuracy of score on the bipolar spectrum diagnostic scale; Bipolar II disorder without borderline personality disorder (left) and borderline personality disorder without bipolar II disorder (right).

The AUC of the MDQ was determined using the total score of part one because using level of impairment data would require multiple ROC curves [13].

The AUCs for the MDQ and BSDS in detecting bipolar II disorder were $0.779(0.674-0.885)$ and 0.778 (0.6620.895 ), respectively (Figures 2 and 3 ).

Similarly, to explore the ability to detect borderline personality disorder with or without co-morbid bipolar II disorder, ROC curves and the AUC (95\% Confidence Interval) of the MDQ (Figures 2 and 4) and BSDS (Figures 3 and 5) were calculated. In detecting borderline personality, the AUCs for the MDQ and BSDS disorder were $0.750(0.597-0.903)$ and 0.787 (0.640-0.934), respectively (Figures 2 and 3). In order to control for the effect of co-morbidity accounting for the diagnostic accuracy of the scales, ROC curves were also calculated for patients with bipolar II disorder but not borderline personality disorder and vice versa. Results remained statistically significant except for the MDQ detecting borderline personality disorder without comorbid bipolar II disorder. Specifically, the AUC for the MDQ was 0.737 (0.605-0.868) for the bipolar II disorder but not borderline personality disorder and 0.691 (0.460-0.923) for borderline personality disorder without comorbid bipolar II disorder. For the BSDS, the AUC was 0.715 (0.566-0.865) for bipolar II disorder but not borderline personality disorder and 0.723 (0.506-0.940) for borderline personality disorder without comorbid bipolar II disorder.

\section{Discussion}

Prior research has suggested that the MDQ and BSDS are useful instruments to detect bipolar disorders among patients with recurrent depressive episodes $[10,11]$. The current study expands on this work by showing that these two scales can detect bipolar disorder among patients with eating disorders. Both the MDQ and BSDS screening scales exhibited similar value in terms of predictive validity in a population of patients presenting to a psychiatric clinic in a tertiary-care setting. Thus, the
MDQ and BSDS offer reasonably comparable diagnostic accuracy in the form of a self-report measure that requires much less time, expertise, and resources to administer than the SCID or Composite International Diagnostic Interview (CIDI). By comparison, the AUC was 0.834 for concordance of bipolar II disorder diagnosis between the SCID and CIDI in a community population [28].

In addition, this study suggests that bipolar disorder and borderline personality disorder can both be detected with moderate accuracy by use of a brief screening instrument, although it was difficult for these scales to detect histrionic personality disorder. Results were similar even after controlling for co-morbidity. This study importantly shows that the two scales can be used as screening tools for borderline personality disorder in a real world setting where eating disorder specialists work. In addition, results showed the assessment of affective instability is useful in terms of bipolar and borderline personality disorder [3], although impulsivity has traditionally been focused in the eating disorder field [4].

Accurate diagnosis and distinction of bipolar disorder and borderline personality disorder is important because of the differing treatment approaches. Psychotherapeutic approaches for these two disorders are very different $[1,4,29]$. Also, pharmacotherapy is a core component of treatment for bipolar disorder, but only adjunctive and symptom-targeted for borderline personality disorder $[1,29]$. These two scales are useful to detect cases that require careful assessment before starting antidepressants, although these scales cannot differentiate between bipolarity and borderline personality disorder.

There are a number of important limitations regarding this study. Sensitivity of these two scales may not be considered sufficiently high using the cut-off point that the original studies suggested. It remains to be known whether the lower sensitivity is due to differences of culture, population, or clinical setting where the participants were recruited. Results are from a single treatment center, and males and patients with bipolar I disorder were not 
included. Results were not analyzed in relationship with eating disorder subtypes (such as restricting or binge/ purging) due to a modest sample size. Assessment was cross-sectional, and careful longitudinal consideration is essential because patients with either bipolar or borderline personality disorder can present with similar symptomatology at a given time [6]. This is especially true in the case of comorbid eating disorder because chaotic eating behaviors and starvation might influence symptomatology [30,31] including dysphoria [32] and anger [9].

\section{Conclusions}

Nonetheless, current results show that these two scales (MDQ and BSDS) are helpful to detect cases that need careful assessment. In addition, we believe these results should encourage further attempts to reconsider the relationship between and symptomatology of bipolar disorder and borderline personality disorder.

\begin{abstract}
Abbreviations
MDQ: Mood Disorder Questionnaire; BSDS: Bipolar Spectrum Diagnostic Scale; EDNOS: Eating disorder not otherwise specified; SCID-I/P: Structured Clinical Interview for DSM-IV; SCID-II: Structured Clinical Interview for DSM-IV Personality Disorders; LR+: Likelihood ratio for a positive test; ROC: Receiver Operating Characteristic; AUC: Area Under the Curve; CIDI: Composite International Diagnostic Interview.
\end{abstract}

\section{Competing interests}

All authors declare that there is no conflict of interest.

\section{Authors' contribution}

All authors contributed to the design of this study. TN and AT drafted the manuscript. All authors contributed to revision of the manuscript. All authors read and approved the final manuscript.

\section{Acknowledgement}

We are thankful to Dr. T. Inada and Dr. T. Tanaka for their permission to use the Japanese versions of the MDQ and BSDS scales.

This work was supported by JSPS KAKENHI Grant Number 24591725.

\section{Author details}

'Department of Neuropsychiatry, Osaka City University Graduate School of Medicine, 1-4-3 Asahimachi, Abunoku, Osaka 545-8585, Japan. ${ }^{2}$ Department of Neuropsychiatry, Hyogo College of Medicine, Nishinomiya, Japan. ${ }^{3}$ Department of Internal Medicine and Department of Psychiatry, University of Michigan, Ann Arbor, USA. ${ }^{4}$ Department of Psychiatry, Leiden University Medical Center, Leiden, the Netherlands.

Received: 31 July 2012 Accepted: 14 February 2013

Published: 25 February 2013

\section{References}

1. Frye MA: Clinical practice. Bipolar disorder--a focus on depression. N Engl J Med 2011, 364(1):51-59.

2. Oldham JM, Gabbard GO, Goin MK, Gunderson J, Soloff P, Spiegel D, Stone M, Phillips KA: Practice guideline for the treatment of patients with borderline personality disorder. American Psychiatric Association. Am J Psychiatry 2001, 158(10 Suppl):1-52.

3. McElroy SL, Kotwal R, Keck PE Jr: Comorbidity of eating disorders with bipolar disorder and treatment implications. Bipolar Disord 2006, 8(6):686-695.

4. Nagata T, Kawarada Y, Kiriike N, Iketani T: Multi-impulsivity of Japanese patients with eating disorders: primary and secondary impulsivity. Psychiatry Res 2000, 94(3):239-250.

5. Paris J: The bipolar spectrum: a critical perspective. Harv Rev Psychiatry 2009, 17(3):206-213.
6. Magill CA: The Boundary Between Borderline Personality Disorder and Bipolar Disorder: Current Concepts and Challenges. Can J Psychiatr 2004 49(8):551-556.

7. Bassett D: Borderline personality disorder and bipolar affective disorder. Spectra or spectre? A review. Aust N Z J Psychiatry 2012, 46(4):327-339.

8. Perugi G, Toni C, Passino MC, Akiskal KK, Kaprinis S, Akiskal HS: Bulimia nervosa in atypical depression: the mediating role of cyclothymic temperament. J Affect Disord 2006, 92(1):91-97.

9. Perugi G, Fornaro M, Akiskal HS: Are atypical depression, borderline personality disorder and bipolar II disorder overlapping manifestations of a common cyclothymic diathesis? World Psychiatr 2011, 10(1):45-51.

10. Hirschfeld RM, Williams JB, Spitzer RL, Calabrese JR, Flynn L, Keck PE Jr, Lewis L, McElroy SL, Post RM, Rapport DJ, et al: Development and validation of a screening instrument for bipolar spectrum disorder: the Mood Disorder Questionnaire. Am J Psychiatry 2000, 157(11):1873-1875.

11. Ghaemi NS, Miller CJ, Berv DA, Klugman J, Rosenquist KJ, Pies RW: Sensitivity and specificity of a new bipolar spectrum diagnostic scale. J Affect Disord 2005, 84(2-3):273-277.

12. Zimmerman M, Galione JN, Chelminski I, Young D, Ruggero CJ: Performance of the Bipolar Spectrum Diagnostic Scale in psychiatric outpatients. Bipolar Disord 2010, 12(5):528-538.

13. Zimmerman M, Galione JN, Ruggero CJ, Chelminski I, Dalrymple K, Young D: Are screening scales for bipolar disorder good enough to be used in clinical practice? Compr Psychiatry 2011, 52(6):600-606.

14. Zimmerman M, Ruggero CJ, Chelminski I, Young D: Psychiatric diagnoses in patients previously overdiagnosed with bipolar disorder. J Clin Psychiatry 2010, 71(1):26-31.

15. Woodside BD, Staab R: Management of psychiatric comorbidity in anorexia nervosa and bulimia nervosa. CNS Drugs 2006, 20(8):655-663.

16. Parker $G$, Fletcher $\mathrm{K}$ : Is bipolar II disorder increasing in prevalence? In Bipolar II disorder: modelling, measuring and managing. 2nd edition. Edited by Parker G. Cambridge, UK; New York: Cambridge University Press; 2012:62-69.

17. Zanarini MC, Reichman CA, Frankenburg FR, Reich DB, Fitzmaurice G: The course of eating disorders in patients with borderline personality disorder: a 10-year follow-up study. Int J Eat Disord 2010, 43(3):226-232.

18. Inada T, Inagaki A, Iwamoto K, Nakatani M, Hori K, Higuchi T: Japanese version of Mood Disorder Questionnaire. In Clinical evaluation of manic disorder by Japanese version of Young Mania Rating Scale (YMRS-J). Tokyo: Jiho; $2005: 35$ (in Japanese).

19. Tanaka T, Koyama T: Rating scales for bipolar disorder: In view of the debate over underdiagnosis and overdiagnosis of bipolar disorder. Rinsho-seishinigaku 2011, 40(3):251-259 (in Japanese).

20. Chu H, Lin CJ, Chiang KJ, Chen CH, Lu RB, Chou KR: Psychometric properties of the Chinese version of the Bipolar Spectrum Diagnostic Scale. J Clin Nurs 2010, 19(19-20):2787-2794.

21. First MB: User's guide for the Structured clinical interview for DSM-IV axis / disorders SCID-l: clinician version. Washington, DC: American Psychiatric Press; 1997.

22. Takahashi S, Kitamura N, Okano T, Tomita T, Kikuchi A: Japanese version of Structured clinical interview for DSM-IV axis I disorders SCID-I. Tokyo: Nihon Hyoronsha; 2003 (in Japanese).

23. First MB, Gibbon M, Spitzer RL, Williams JBW, Benjamin L: Structured clinical interview for DSM-IV axis II personality disorders, SCID-II. Washington, DC American Psychiatric Press; 1997.

24. Takahashi S, Ohsone A: Japanese version of SCID-II (Structured Clinical Interview for DSM-IV Axis II Personality Disorders). Tokyo: Igaku-Shoin; 2002 (in Japanese).

25. Nagata T, Kawarada Y, Ohshima J, Iketani T, Kiriike N: Drug use disorders in Japanese eating disorder patients. Psychiatry Res 2002, 109(2):181-191.

26. Joyce PR, Luty SE, McKenzie JM, Mulder RT, McIntosh W, Carter FA, Bulik CM, Sullivan PF: Bipolar II disorder: personality and outcome in two clinical samples. Aust N Z J Psychiatry 2004, 38(6):433-438.

27. Akobeng AK: Understanding diagnostic tests 2: likelihood ratios, pre- and post-test probabilities and their use in clinical practice. Acta Paediatr 2007, 96(4):487-491.

28. Kessler RC, Akiskal HS, Angst J, Guyer M, Hirschfeld RM, Merikangas KR, Stang PE: Validity of the assessment of bipolar spectrum disorders in the WHO CIDI 3.0. J Affect Disord 2006, 96(3):259-269.

29. Gunderson JG: Clinical practice. Borderline personality disorder. N Engl J Med 2011, 364(21):2037-2042. 
30. Lilenfeld LR: Personality and temperament. Curr Top Behav Neurosci 2011, 6:3-16.

31. Renaud S, Corbalan F, Beaulieu S: Differential diagnosis of bipolar affective disorder type II and borderline personality disorder: analysis of the affective dimension. Compr Psychiatry 2012, 53(7):952-961.

32. Kaye W: Eating disorders: hope despite mortal risk. Am J Psychiatry 2009, 166(12):1309-1311.

doi:10.1186/1471-244X-13-69

Cite this article as: Nagata et al.: Using the mood disorder questionnaire and bipolar spectrum diagnostic scale to detect bipolar disorder and borderline personality disorder among eating disorder patients. BMC Psychiatry 2013 13:69.

\section{Submit your next manuscript to BioMed Central and take full advantage of:}

- Convenient online submission

- Thorough peer review

- No space constraints or color figure charges

- Immediate publication on acceptance

- Inclusion in PubMed, CAS, Scopus and Google Scholar

- Research which is freely available for redistribution 